\title{
Can Mental Health Apps Be Effective for Depression, Anxiety, and Stress During a Pandemic?
}

\author{
Robert L. Longyear ${ }^{1}$ and Kostadin Kushlev ${ }^{1}$ \\ ${ }^{1}$ Department of Psychology, Georgetown University
}

\begin{abstract}
Author Note
We declare no conflicts of interest. No human subjects were involved in the research conducted by the authors. Correspondence concerning this paper should be addressed to Robert L. Longyear, r1157@georgetown.edu
\end{abstract}




\begin{abstract}
Covid-19 is poised to exacerbate the global mental health crisis as social isolation, unemployment, and economic recession are risk factors for negative mental health consequences. Since the need for social distancing can make face-to-face services less accessible, people may turn to mental health apps as an accessible and inexpensive solution. But are the claims about the efficacy of these apps supported by evidence? We identified 19 published articles on PubMed of peer-reviewed randomized clinical trials of mental health apps focused on stress, anxiety, and depression. Despite some evidence for the effectiveness of mental health apps, it remains unclear how effective these apps are compared to standard of care. Populations studied so far also lack diversity, making it difficult to generalize any benefits to racial minorities and low-income individuals - the very people who have been most negatively impacted by the Covid-19 pandemic and traditionally have higher barriers to mental health services. While apps are not a substitute for face-to-face therapy, further evidence is warranted given their potential for delivering scalable, low-cost care to patients. We call for regulatory oversight as a means to ensure mental health apps demonstrate safety and effectiveness prior to marketing.
\end{abstract}

Keywords: Mental Health, mHealth, Depression, Anxiety, Stress, Mental Health Access, Rural Health, Telehealth, Health Policy

Clinical Impact Statement: Mental health apps are increasingly available to consumers/patients for depression, stress, and anxiety. Early evidence suggests that those apps studied have a positive impact on these conditions, but further research and regulatory oversight is needed before apps should be recommended to patients. 


\section{Can Mental Health Apps Be Effective for Depression, Anxiety, and Stress During a Pandemic?}

Long before the Covid-19 pandemic, a mental health pandemic has been afflicting people around the globe: mental health disorders have risen by more than 30\% since 1990 (James et al., 2018). Covid-19 is poised to exacerbate the global mental health crisis as social isolation (Hawryluck et al., 2004), unemployment (Anusic et al., 2014), and economic recession (Diener et al., 2015) are all well-documented risk factors for mental health conditions (Maunder et al., 2003).

Less than one out of three people with mental health disorders receive treatment, and among those that do, a smaller proportion receives adequate care (Olfson et al., 2016). Populations with limited access to mental health services (Green et al., 2020) prior to Covid-19 are the same under-resourced populations that have been disproportionately affected by the pandemic (Laurencin \& McClinton, 2020). And while social distancing has proven an invaluable tool for controlling the spread of Covid-19 (Ferguson et al., 2020), social distancing can also entail less access to the standard of care in-person services. Can stand-alone mental health apps provide viable alternative during the Covid-19 pandemic?

In 2019, a review found hundreds of mental health apps were available for download on both Android and iOS app stores (see Figure 1). The same review concluded that many of those apps claim to be have a basis in established positive and clinical psychological principles (Larsen et al., 2019). Clinicians have also expressed interest in supplementing care through mobile apps (Boudreax et al., 2014). Recent meta-analyses have found that self-contained smartphone-based interventions have moderate effects on both anxiety (Firth et al., 2017a) and depression (Firth et al., 2017b). 
Despite the promising evidence, the vast majority of app developers make claims that are unsubstantiated by direct evidence for their app's efficacy. The UK National Health Service Health Apps Library, for example, found that only 15\% of apps provided evidence of effectiveness (Leigh \& Flatt, 2015). And a 2016 study of available depression apps found that $38 \%$ of app store descriptions included wording related to the app's effectiveness, but only $2.6 \%$ provided evidence to substantiate claims (Larsen et al., 2016). In an unregulated market, such unsubstantiated claims may be the only source of consumer information. Fueling this marketing push is a significant growth in venture capital investing in mental health apps (Shah \& Obianuju, 2020). This presents a potential risk to consumers, especially considering that both outpatient psychiatric patients (Torous et al., 2014) and consumers in general (Proudfoot et al., 2010) are open to receiving care via a mobile app. As mental health apps become ever more widely adopted, it is essential to continue to examine their efficacy and effectiveness using the gold standards in clinical science.

\section{Focus of the Present Review}

As the Covid-19 pandemic continues to impact mental health, their scalability, accessibility, and cost-effectiveness make mental health apps a potentially valuable tool within the greater mental health intervention toolbox. Thus, we sought to evaluate the state of evidence, broadly, for mental health apps. Given the clinical effectiveness claims of these apps, we only included randomized clinical trials (RCTs) in our review. Given consumer interest in mental health apps and a lack of clear regulatory oversight, we further focus on apps that are intended for use directly by consumers and patients rather than clinicians. 


\section{Methods}

To better understand the evidence for the efficacy and effectiveness of mental health apps, we performed a keyword search (i.e., mental, health, app, randomized, clinical, trial) of PubMed for RCTs of mental health apps focused on stress, anxiety, and depression. These three conditions are areas of heavy focus for mental health apps (Larsen et al., 2016). Our search for peer-reviewed, published studies yielded 94 results on PubMed. After an abstract review, we included clinical trials that assessed efficacy and used self-contained app-based interventions, but not telepsychology or technology-facilitated standard-of-care modalities (e.g., therapist video conference, or prescription management).

\section{Results}

We identified 19 completed and published clinical trials that fit our criteria (see Table 1). In 18 of those RCTs, the app-based interventions significantly reduced self-reported stress, anxiety, and/or depression symptoms (e.g., Arean, 2016; Graham, 2020; Huberty, 2019; Matani, 2017). However, the evidence is plagued by several common methodological issues in the studies we reviewed. First, the vast majority of studies included a three-month follow-up period, it remains unknown whether the observed effects persist in the long term. Second, the RCTs reviewed here relied primarily on samples that are overwhelmingly white, female, and younger. Given this dearth of RCT evidence in diverse populations, it remains unclear whether selfcontained app-based interventions will be as effective for people of color, males, and older individuals in the population. We found only one study that specifically sought to recruit from Hispanic/Latinx populations. Positive outcomes were sustained across all sub-populations studied, but the study found that long-term engagement among Hispanic/Latinx participants to be lower than among Non-Hispanic/Latinx participants (Pratap et al., 2018). Furthermore, the 
relative lack of non-white populations in the studies we reviewed calls into question the very premise that mobile apps are a viable way to reach populations with typically poor access or uptake of traditional mental health services.

Third, the majority of RCTs in this review obtained samples below 200 participants (Table 1). The marketing of apps to consumers as potential treatments of stress, anxiety, or depression should merit a higher burden of clinical evidence on par with medical standards of care. Before psychiatric drugs, for example, can be approved and marketed to consumers, the FDA requires efficacy clinical trials with at least 300, to as many as 3,000, participants (US Food and Drug Administration, 2020). While apps are not equivalent to drugs, apps may be adopted by high-risk patients with mental illness; without sufficient sample size, it is difficult to gauge potential low frequency adverse events of app utilization, such as self-harm or suicide rates.

Finally, another weakness in the studies we reviewed is that none directly compared appbased intervention with the standard of care. Most studies employed a waitlist-control design, whereby the efficacy of an app-based intervention was compared to no treatment. Five studies included multiple intervention groups wherein one group acted as a placebo (typically, information-only control); these studies suggest that the active elements of mental health apps contribute to their beneficial effects. Still, future research needs to include true placebo groups, whereby participants use a similar app with engaging activities that are not designed to improve mental health (e.g., a fun gaming app). Future research also needs to directly compare both the efficacy and effectiveness of mental health apps with standard of care, such as traditional faceto-face therapy or medications. Even though mental health apps will never replace traditional face-to-face therapy, their low-cost and convenience could be leveraged to reach more people 
who need help managing their mental health symptoms; further evaluation is needed to understand the comparative effects of mental health apps.

One of the studies we found overcomes some of the issues pervading the larger body of evidence (Graham et al., 2020). In a sample of 146 primary care patients, the study established the efficacy of a mobile app platform in reducing symptoms of anxiety and depression over an eight-week treatment, with sustained effects at a sixteen-week follow-up. Though men were underrepresented, comprising less than $20 \%$ of participants, the sample was racially diverse with Black people comprising a third of the sample. At $\$ 42,000$, the sample's median household income was substantially lower than that of the general U.S. population $(\$ 68,703$; Semega et al., 2020), and almost half of the sample did not have a bachelor's degree. The efficacy of this appbased intervention in this sample suggests that mobile apps can be a viable solution for providing symptom alleviation to populations of lower socioeconomic status.

\section{Discussion}

The RCT evidence we reviewed here suggests that mental health apps can be beneficial to some people. It remains unclear, however, how effective mental health apps are in the context of the larger clinical toolbox for mental health or in specific subpopulations.

Despite these promising results, the lack of clear regulatory oversight sets the stage for widespread and unchecked marketing of unsubstantiated claims. Without a clear pre-market or post-market monitoring process, many mental health apps are available to consumers with little understanding of their effectiveness or the incidence of adverse events. Lack of regulatory oversight can also contribute to a failure to report studies with no, or adverse, effects of appbased interventions; such publication bias can only be exacerbated by the commercial interests of app developers. Clinicaltrials.gov is a resource used to track the outcomes from RCTs by 
regulators and represents a potential remedy for these publication bias issues in this area of research. This registry also includes information on IRB-approval, another important oversight mechanism. However, a recent review of clinicaltrials.gov by DeVito and colleagues, suggests that a significant portion of registered clinical trials do not report results (2020). Given the lack of regulatory oversight, there exists the potential for nonsignificant or adverse effects to go unreported. A well-defined pathway for pre-market regulatory approval should be outlined for mental health apps due to the vulnerability of the patient populations that they target.

Mental health apps have gained attention during Covid-19 due to their scalability and accessibility. Initial evidence supports their efficacy, but not yet their effectiveness, compared to no treatment at all, but mostly in populations that already have access to care. During the Covid19 pandemic, these mental health apps do have the potential to provide benefit given barriers to typical care. Because the current mental health system is already a two-tiered system with significant barriers to access for certain populations, however, it is possible that these apps only perpetuate the disparities in mental health care as those with least access to current standards of care are left to rely on apps. Thus, it is imperative that the effectiveness of these apps is understood as compared to standard of care specifically in populations with disproportionally lower access to and utilization of existing mental health services. More broadly, the claims made by app developers deserve more regulatory oversight to protect consumers and ensure rigor of research and reporting.

Word Count: 1682 


\section{References}

Anusic, I., Yap, S. C. Y., \& Lucas, R. E. (2014). Testing set-point theory in a Swiss national sample: Reaction and adaptation to major life events. Social Indicators Research, 119, 1265-1288. http://dx.doi.org/10.1007/ s11205-013-0541-2

Arean, P., Hallgren, K., Jordan, J., Gazzaley, A., Atkins, D., Heagerty, P., \& Anguera, J. (2016). The use and effectiveness of mobile apps for depression: Results from a fully remote clinical trial. Journal of Medical Internet Research, 18(12), e330. https://doi.org/10.2196/jmir.6482

Bakker, D., Kazantzis, N., Rickwood, D., \& Rickard, N. (2018). A randomized controlled trial of three smartphone apps for enhancing public mental health. Behaviour Research and Therapy, 109, 75-83. https://doi.org/10.1016/j.brat.2018.08.003

Boudreaux, E.D., Waring, M.E., Hayes, R.B., Sadasivam, R.S., Mullen, S., \& Pagoto, S. (2014). Evaluating and selecting mobile health apps: strategies for healthcare providers and healthcare organizations. Translational Behavioral Medicine, 4(4), 363-371. https://doi.org/10.1007/s13142-014-0293-9

Bush, N. E., Smolenski, D. J., Denneson, L. M., Williams, H. B., Thomas, E. K., \& Dobscha, S. K. (2017). A Virtual Hope Box: Randomized Controlled Trial of a Smartphone App for Emotional Regulation and Coping With Distress. Psychiatric Services, 68(4), 330-336. https://doi.org/10.1176/appi.ps.201600283

Champion, L., Economides, M., \& Chandler, C. (2018). The efficacy of a brief app-based mindfulness intervention on psychosocial outcomes in healthy adults: A pilot randomised controlled trial. PLOS ONE, 13(12), e0209482. https://doi.org/10.1371/journal.pone.0209482 
Cuijpers, P., Van Straten, A.,Warmerdam, L., \& Smits N. (2008). Characteristics of effective psychological treatments of depression: A metaregression analysis. Psychotherapy Res.,18(2):225-236. https://doi.org/10.1080/10503300701442027

DeVito, N. J., Bacon, S., \& Goldacre, B. (2020). Compliance with legal requirement to report clinical trial results on ClinicalTrials.gov: A cohort study. The Lancet, 395(10221), 361369. https://doi.org/10.1016/s0140-6736(19)33220-9

Diener, E., Oishi, S., \& Lucas, R. E. (2015). National accounts of subjective well-being. American Psychologist, 70, 234-242. http://dx.doi.org/ 10.1037/a0038899

Ferguson, N.M., Laydon, D., Nedjati-Gilani, G. et al. (2020). Impact of non-pharmaceutical interventions (NPIs) to reduce COVID-19 mortality and healthcare demand. Imperial College London. https://doi.org/10.25561/77482.

Firth, J., Torous, J., Nicholas, J., Carney, R., Rosenbaum, S., \& Sarris, J. (2017a). Can smartphone mental health interventions reduce symptoms of anxiety? A meta-analysis of randomized controlled trials. Journal of Affective Disorders, 218,15-22. https://doi.org/10.1016/j.jad.2017.04.046

Firth, J., Torous, J., Nicholas, J., Carney, R., Pratap, A., Rosenbaum, S., \& Sarris, J. (2017b). The efficacy of smartphone-based mental health interventions for depressive symptoms: A meta-analysis of randomized controlled trials. World Psychiatry, 16(3):287-298. https://doi.org/10.1002/wps.20472

Fitzpatrick, K. K., Darcy, A., \& Vierhile, M. (2017). Delivering Cognitive Behavior Therapy to Young Adults With Symptoms of Depression and Anxiety Using a Fully Automated Conversational Agent (Woebot): A Randomized Controlled Trial. JMIR Mental Health, 4(2), e19. https://doi.org/10.2196/mental.7785 
Graham, A., Greene, C., Kwasny, M., Kaiser, S., Lieponis, P., Powell, T., \& Mohr, D. (2020). Coached Mobile App Platform for the Treatment of Depression and Anxiety Among Primary Care Patients. JAMA Psychiatry. https://doi.org/10.1001/jamapsychiatry.2020.1011

Green, J. G., Mclaughlin, K. A., Fillbrunn, M., Fukuda, M., Jackson, J. S., Kessler, R. C., . . . Alegría, M. (2020). Barriers to mental health service use and predictors of treatment drop out: Racial/Ethnic variation in a population-based study. Administration and Policy in Mental Health and Mental Health Services Research, 47(4), 606-616. https://doi.org/10.1007/s10488-020-01021-6

Hantsoo, L., Criniti, S., Khan, A., Moseley, M., Kincler, N., Faherty, L. J., Epperson, C. N., \& Bennett, I. M. (2018). A Mobile Application for Monitoring and Management of Depressed Mood in a Vulnerable Pregnant Population. Psychiatric Services, 69(1), 104107. https://doi.org/10.1176/appi.ps.201600582

Harrer, M., Adam, S. H., Fleischmann, R. J., Baumeister, H., Auerbach, R., Bruffaerts, R., Cuijpers, P., Kessler, R. C., Berking, M., Lehr, D., \& Ebert, D. D. (2018). Effectiveness of an Internet- and App-Based Intervention for College Students With Elevated Stress: Randomized Controlled Trial. Journal of Medical Internet Research, 20(4), e136. https://doi.org/10.2196/jmir.9293

Hawryluck, L., Gold, W. L., Robinson, S., Pogorski, S., Galea, S., \& Styra, R. (2004). SARS control and psychological effects of quarantine, Toronto, Canada. Emerging Infectious Diseases, 10(7), 1206-1212. https://doi.org/10.3201/eid1007.030703)

Huberty, J., Green, J., Glissmann, C., Larkey, L., Puzia, M., \& Lee, C. (2019). Efficacy of the mindfulness meditation mobile app "Calm" to reduce stress among college students: 
Randomized controlled trial. JMIR Mhealth And Uhealth, 7(6), e14273. https://doi.org/10.2196/14273

Hur, J.-W., Kim, B., Park, D., \& Choi, S.-W. (2018). A Scenario-Based Cognitive Behavioral Therapy Mobile App to Reduce Dysfunctional Beliefs in Individuals with Depression: A Randomized Controlled Trial. Telemedicine and E-Health, 24(9), 710-716. https://doi.org/10.1089/tmj.2017.0214

Hwang, W. J., \& Jo, H. H. (2019). Evaluation of the Effectiveness of Mobile App-Based StressManagement Program: A Randomized Controlled Trial. International Journal of Environmental Research and Public Health, 16(21), 4270. https://doi.org/10.3390/ijerph16214270

James, S. L., Abate, D., Abate, K. H., Abay, S. M., Abbafati, C., Abbasi, N., ... Murray, C. J. L. (2018). Global, regional, and national incidence, prevalence, and years lived with disability for 354 Diseases and Injuries for 195 countries and territories, 1990-2017: A systematic analysis for the Global Burden of Disease Study 2017. The Lancet, 392(10159), 1789-1858. https://doi.org/10.1016/S0140-6736(18)32279-7

Larsen, M., Huckvale, K., Nicholas, J., Torous, J., Birrell, L., Li, E., \& Reda, B. (2019). Using science to sell apps: Evaluation of mental health app store quality claims. Npj Digital Medicine, 2(1). https://doi.org/10.1038/s41746-019-0093-1

Larsen, M., Nicholas, J., \& Christensen, H. (2016). Quantifying app store dynamics: Longitudinal tracking of mental health apps. JMIR Mhealth And Uhealth, 4(3), e96. https://doi.org/10.2196/mhealth.6020 
Laurencin, C. T., \& McClinton, A. (2020). The COVID-19 pandemic: A call to action to identify and address racial and ethnic disparities. Journal of Racial and Ethnic Health Disparities, 7(3), 398-402. https://doi.org/10.1007/s40615-020-00756-0

Leigh S, \& Flatt S. (2015). App-based psychological interventions: friend or foe? Evidence Based Mental Health. 18(4):97-99.

Lüdtke, T., Pult, L. K., Schröder, J., Moritz, S., \& Bücker, L. (2018). A randomized controlled trial on a smartphone self-help application (Be Good to Yourself) to reduce depressive symptoms. Psychiatry Research, 269, 753-762.

https://doi.org/10.1016/j.psychres.2018.08.113

Mantani, A., Kato, T., Furukawa, T., Horikoshi, M., Imai, H., \& Hiroe, T. et al. (2017). Smartphone cognitive behavioral therapy as an adjunct to pharmacotherapy for refractory depression: Randomized controlled trial. Journal Of Medical Internet Research, 19(11), e373. https://doi.org/10.2196/jmir.8602

Maunder, R., Hunter, J., Vincent, L., Bennett, J., Peladeau, N., Leszcz, M., ... Mazzulli, T. (2003). The immediate psychological and occupational impact of the 2003 SARS outbreak in a teaching hospital. Canadian Medical Association journal, 168(10), 12451251.

Moberg, C., Niles, A., \& Beermann, D. (2019). Guided Self-Help Works: Randomized Waitlist Controlled Trial of Pacifica, a Mobile App Integrating Cognitive Behavioral Therapy and Mindfulness for Stress, Anxiety, and Depression. Journal of Medical Internet Research, 21(6), e12556. https://doi.org/10.2196/12556

Mohr, D. C., Schueller, S. M., Tomasino, K. N., Kaiser, S. M., Alam, N., Karr, C., Vergara, J. L., Gray, E. L., Kwasny, M. J., \& Lattie, E. G. (2019). Comparison of the Effects of 
Coaching and Receipt of App Recommendations on Depression, Anxiety, and Engagement in the IntelliCare Platform: Factorial Randomized Controlled Trial. Journal of Medical Internet Research, 21(8), e13609. https://doi.org/10.2196/13609

Olfson, M., Blanco, C., \& Marcus, S.C. (2016). Treatment of adult depression in the United States. JAMA Intern Medicine, 176(10), 1482-1491. https://doi.org/10.1001/ jamainternmed.2016.5057

Pratap, A., Renn, B. N., Volponi, J., Mooney, S. D., Gazzaley, A., Arean, P. A., \& Anguera, J. A. (2018). Using mobile apps to assess and treat depression in hispanic and latino populations: Fully remote randomized clinical trial. Journal of Medical Internet Research, 20(8), e10130. https://doi.org/10.2196/10130

Proudfoot, J., Parker, G., Hadzi Pavlovic, D., Manicavasagar, V., Adler, E., \& Whitton, A. (2010). Community attitudes to the appropriation of mobile phones for monitoring and managing depression, anxiety, and stress. Journal of Medical Internet Research, 12(5), 64. https://doi.org/10.2196/jmir.1475

Roepke, A. M., Jaffee, S. R., Riffle, O. M., McGonigal, J., Broome, R., \& Maxwell, B. (2015). Randomized Controlled Trial of SuperBetter, a Smartphone-Based/Internet-Based SelfHelp Tool to Reduce Depressive Symptoms. Games for Health Journal, 4(3), 235-246. https://doi.org/10.1089/g4h.2014.0046

Shafran, R., Gyani, A., Rostron, J., Allen, S., Myles-Hooton, P., Allcott-Watson, H., \& Rose, S. (2018). Translating the Intention to Seek Treatment into Action: Does Symptom Monitoring Make a Difference? Results from a Randomized Controlled Trial. Behavioural and Cognitive Psychotherapy, 47(1), 114-128. https://doi.org/10.1017/s1352465818000498 
Shah, R.N., Berry, O.O. (2020). The rise of venture capital investing in mental health. JAMA Psychiatry. Online. https://doi.org/10.1001/jamapsychiatry.2020.2847

Semega, J., Melissa K., Emily, S.A., \& Creamer, J.F. (2020). U.S. Census Bureau, Current Population Reports, P60-270, Income and Poverty in the United States: 2019, U.S. Government Publishing Office.

Torous J., Chan S.R., Yee-Marie T.S., Behrens J., Mathew I., Conrad E.J., et al. (2014). Patient smartphone ownership and interest in mobile apps to monitor symptoms of mental health conditions: A survey in four geographically distinct psychiatric clinics. JMIR Mental Health,1(1), 5. https://dx.doi.org/10.2196\%2Fmental.4004

U.S. Food and Drug Administration. (2020). The Drug Development Process. Online. 


\section{Figures \& Tables}

Figure 1

Number of Depression and Anxiety Apps Identified on App Stores

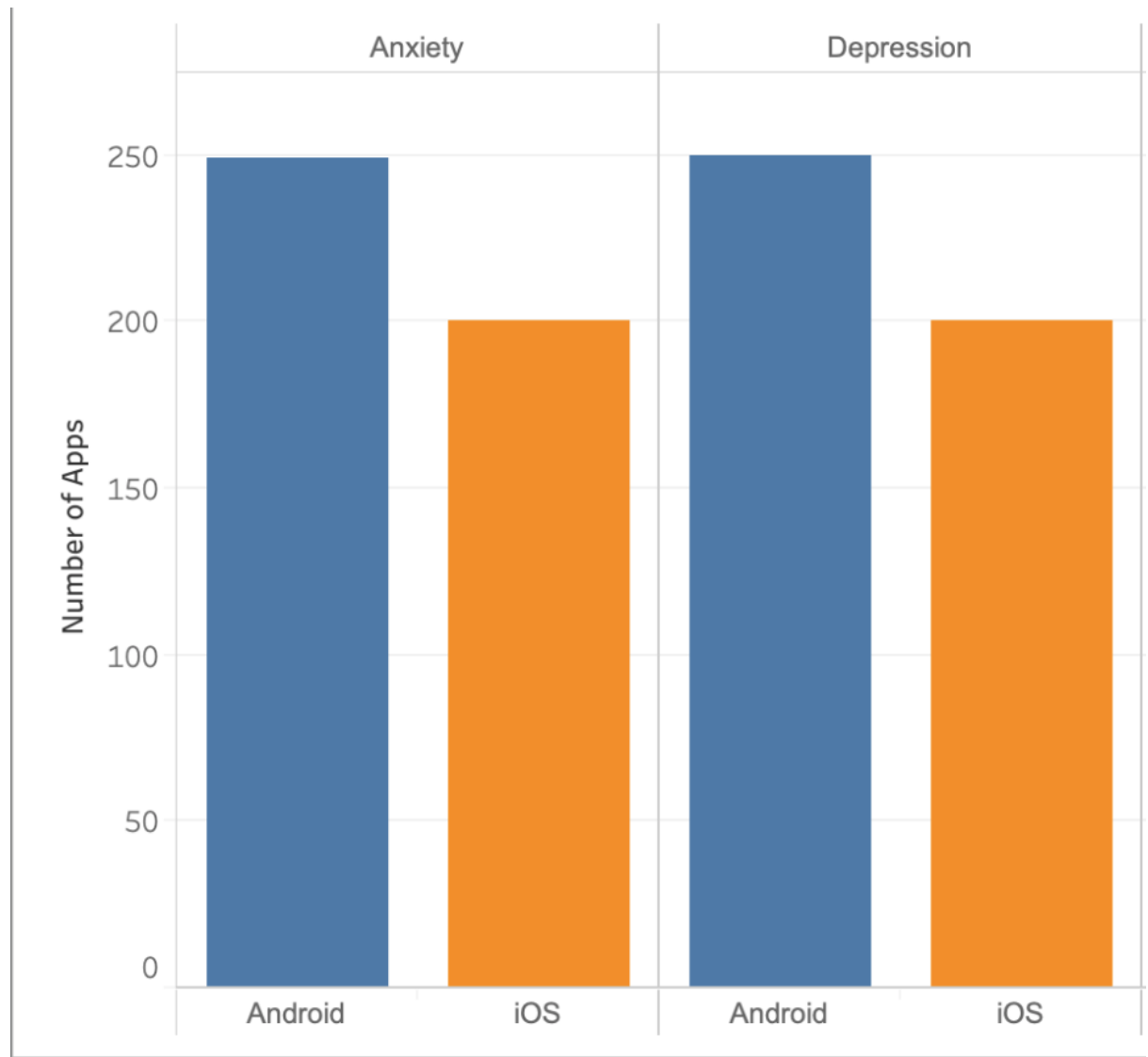

Note. Number of applications available for consumer download on major app stores via an app store search. There is overlap between apps on both app stores. Data from: Larsen, M., Huckvale, K., Nicholas, J., Torous, J., Birrell, L., Li, E., \& Reda, B. (2019). Using science to sell apps: Evaluation of mental health app store quality claims. Npj Digital Medicine, 2(1). doi: 10.1038/s41746-019-0093-1 
Table 1

Summary of 19 randomized controlled trials testing the effects of mental health apps for depression, anxiety, and/or stress

\begin{tabular}{ccccc}
\hline Study & Year & $\begin{array}{c}\text { Sample } \\
\text { Size }\end{array}$ & $\begin{array}{c}\text { Comparison } \\
\text { Condition(s) }\end{array}$ & Effects of Mental Health App \\
\hline Roepke et al. & 2015 & 283 & Waitlist Control & Improvement in depression \\
Arean et al. & 2016 & 626 & Information Control & Improvement in depression \\
Bush et al. & 2017 & 118 & Information Control & Improvement in coping with unpleasant emotions \\
Mantani et al. & 2017 & 164 & Negative Control & Improvement in depression \\
Fitzpatrick et al. & 2017 & 70 & Information Control & Improvements in anxiety and depression \\
Bakker et al. & 2018 & 226 & Waitlist Control & Improvement in depression, no effect on anxiety \\
Harrer et al. & 2018 & 150 & Waitlist Control & Improvements in stress, anxiety, and depression \\
Champion et al. & 2018 & 38 & Waitlist Control & Improvement in stress \\
Hantsoo et al. & 2018 & 72 & Patient Portal Control & Improvement in depression \\
Hur et al. & 2018 & 34 & Mood Chart Control & Improvements in anxiety and depression \\
Lüdtke et al. & 2018 & 90 & Waitlist Control & Improvement in depression \\
Pratap et al. & 2018 & 348 & Information Control & Improvement in depression \\
Huberty et al. & 2019 & 88 & Waitlist Control & Improvement in stress \\
Moberg et al. & 2019 & 500 & Waitlist Control & Improvement in stress, anxiety, and depression \\
Hwang et al. & 2019 & 56 & Negative Control & Improvement effect in stress \\
Mohr et al. & 2019 & 301 & App Control & Improvements in anxiety and depression \\
Shafran et al. & 2019 & 306 & Information Control & Increase in treatment seeking \\
Graham et al. & 2020 & 146 & Waitlist Control & Improvements in depression and anxiety
\end{tabular}

Note. Results obtained from PubMed key work search using: “mental," “health," “app," “randomized," “clinical," "trial.” This search yielded 94 results which were screened for applications focused on consumers working independently from the clinician i.e. a self-contained application. Inclusion also required studies look at outcome measures related to depression, stress, and anxiety. 